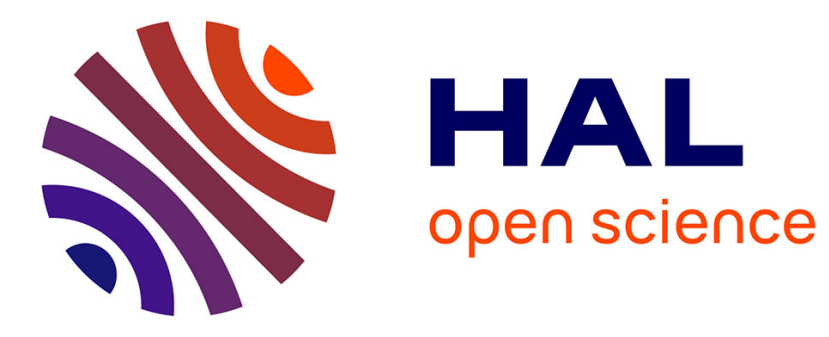

\title{
A circuit-breaker use-case operated by a humanoid in aircraft manufacturing
}

Anastasia Bolotnikova, Kevin Chappellet, Antonio Paolillo, Adrien Escande, Gholamreza Anbarjafari, Adolfo Suarez-Roos, Patrice Rabaté, Abderrahmane Kheddar

\section{To cite this version:}

Anastasia Bolotnikova, Kevin Chappellet, Antonio Paolillo, Adrien Escande, Gholamreza Anbarjafari, et al.. A circuit-breaker use-case operated by a humanoid in aircraft manufacturing. CASE: Conference on Automation Science and Engineering, Aug 2017, Xi'an, China. pp.15-22, 10.1109/COASE.2017.8256069 . hal-01565060

\section{HAL Id: hal-01565060 \\ https://hal.science/hal-01565060}

Submitted on 19 Jul 2017

HAL is a multi-disciplinary open access archive for the deposit and dissemination of scientific research documents, whether they are published or not. The documents may come from teaching and research institutions in France or abroad, or from public or private research centers.
L'archive ouverte pluridisciplinaire HAL, est destinée au dépôt et à la diffusion de documents scientifiques de niveau recherche, publiés ou non, émanant des établissements d'enseignement et de recherche français ou étrangers, des laboratoires publics ou privés. 


\title{
A circuit-breaker use-case operated by a humanoid in aircraft manufacturing
}

\author{
Anastasia Bolotnikova ${ }^{1,2}$, Kévin Chappellet ${ }^{2}$, Antonio Paolillo ${ }^{2}$, Adrien Escande ${ }^{3}$, Gholamreza Anbarjafari ${ }^{1,4}$, \\ Adolfo Suarez-Roos ${ }^{5}$, Patrice Rabaté ${ }^{5}$ and Abderrahmane Kheddar ${ }^{3,2}$
}

\begin{abstract}
Automation of large-scale aircraft manufacturing with wheeled or embedded platforms requires costly changes of the manufacturing process and the environment. Humanoid robots could address this issue. We present a use-case of HRP-4 humanoid operating circuit-breakers. We show the feasibility of using visual feedback and force control in an integrated and unified multi-contact and multimodal task space quadratic programming (QP) whole-body control framework to enable HRP -4 to perform the task. We discuss the experimental results and outline the limitations of the current platform design and control implementation.
\end{abstract}

\section{INTRODUCTION}

Humanoid robots (humanoids) are gaining more maturity in terms of hardware and embedded software. Each year we witness the improvement of an existing series and the revelation of new platforms. So far, humanoids' developments have been confined to research, and there is still much to do in several specific aspects, e.g. robust dynamic walking, multi-contact behaviors or those common to general robotics research. As humanoids are getting close to well fulfil their functionalities in terms of locomotion and manipulation it is time to anticipate what they could be used for.

The first revealed applications of humanoids are oriented toward entertainments, e.g. [1], [2]. Some applications target domotic assistance for frail persons ${ }^{1}$, where a humanoid can offer assistance services [3]. Recently the DARPA robotic challenge $^{2}$ opened potential applications in rescue and disaster operations. Yet the contest brought into light the gap that remains to overcome in order to efficiently deploy such a technology outdoor and achieve complex tasks such as driving [4] or ladder climbing [5]. During the two previous years, we focused our efforts on applications of humanoids in manufacturing. There has been clear expression of the requirements by Airbus Group Innovation (AGI) for having humanoids operate in aerospace large-scale manufacturing and assembly lines, see section II.

In the frame of two on-going projects with AGI, this paper discusses the integration of our planning and control software components and knowledge in a first use-case consisting in operating circuit-breakers (pulling or pushing switches laid out in a panel), section II. This operation is found in the

1- University of Tartu, Estonia

2- CNRS-UM LIRMM, Interactive Digital Humans, Montpellier, France

3- CNRS-AIST Joint Robotics Laboratory, UMI3218/RL, Tsukuba, Japan

4- Department of Electrical and Electronic Engineering, HKU, Turkey

5- Airbus Group Innovation Center, Suresnes, France

${ }^{1}$ http://http://www.projetromeo.com/

${ }^{2}$ http://www.theroboticschallenge.org/ manufacturing process and at the ground-testing operations of both airplanes and helicopters. We focus on demonstrating the feasibility of our multi-contact planning and control tools [6][7][5] in this use-case. Our original contribution stands as follows:

- adding two functionalities to our whole-body quadratic programming (QP) control framework: we provide a multi-modal integration of force and visual servoing tasks in the framework together with other tasks such as fixed contacts, reaching, center-of-mass positioning, etc. (section III);

- we extend our multi-contact planning to consider creating closed-kinematic chains, when possible, to fulfil the requirement of operating push/pull button task so that the humanoid equilibrium and pulling forces can be increased (section IV);

- we demonstrate the circuit breaker checking task with the HRP-4 humanoid and discuss performances and limitations of the current set-up to be improved in nextstage developments (section V).

Because of its primacy, we highlight the ambition behind deploying manufacturing humanoids in airliner assembly lines.

\section{HumANOID TECHNOLOGY IN AIRCRAFT MANUFACTURING}

At the Airbus airliners assembly lines, most of the assembly or system installation operations are achieved manually, especially those that have to be performed inside the aircraft. Robotic solutions have not found their way into all areas of aeronautical assembly lines because of safety, accessibility, weight, and the complexity of the operations to be performed. AGI developed a first prototype of a collaborative robot. This cobot, mainly composed of an intrinsically safe robot arm (Kuka LWR) mounted on an omnidirectional wheeled platform, is capable of performing some dedicated tasks inside an aircraft fuselage while sharing its workspace with several human workers. It is able to move on relatively flat floors, and to avoid obstacles.

Yet, there are still many manufacturing zones that cannot be reached by a wheeled cobot, e.g. in the cargo areas, cockpit, aisles. This is precisely where legged robots may be able to provide a more complete manufacturing solution, see Fig. 1. Furthermore, a wheeled cobot is confined to a given floor because it is not capable of autonomously moving to another platform within the same aircraft. While the installation of elevators could be a solution, it is costly 


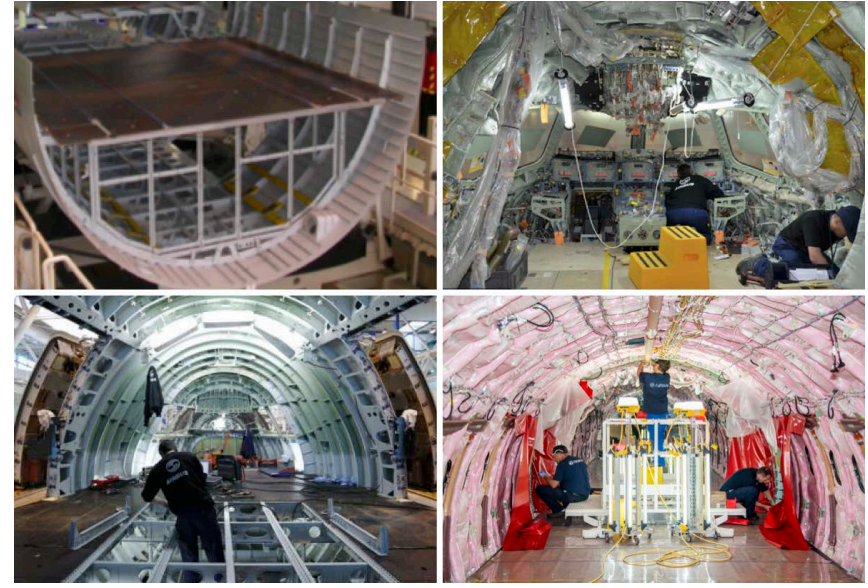

Fig. 1: Example of shop floors and environments (from left to right, top to down): the cargo area, the cockpit, the upper-area in two different phases of the assembly/installation. Notice the postures of the workers. A humanoid is expected to have such accessibility capabilities.

and cannot be deployed everywhere. On the other hand, a humanoid platform would be free of this constraint since it can use the stairs already in place for human workers [8].

If we want to increase the level of automation, in order to make the shop floor productive and keep manufacturing plants in developed countries, we have to devise robots capable of:

- working in such confined, cluttered, and rugged areas;

- performing varying operations with standard manual tools (like humans);

- moving autonomously through the manufacturing environment designed for humans.

The motivation behind using humanoid technology is not solely driven by a wish to increase the level of automation. In fact, we mainly aim at addressing human workers health and safety issues. The goal is to use humanoids to perform "nonadded value tasks": repetitive tasks where the experience and intelligence of the operator are not put to use and where boredom can lead to mistakes, tasks presenting health risks such as anti-corrosion painting (highly toxic) or cleaning (after use of solvents, metallic dirt after drilling...) that are usually performed in confined spaces.

If safety requirements are met, a humanoid could perform a number of operations, such as:

- Accurate assembly operations, e.g. riveting, drilling and screwing using manual or semi-automatic hand tools;

- Cleaning and painting operations;

- System installation: electrical harness installation, connector plugging, etc.

Humanoids could also be used to perform other nonadded value tasks such as conveying equipment or tools to highly qualified operators: hundreds of thousand parts and tools are transported and manipulated by operators for each aircraft. By unburdening highly qualified operators from such boring tasks, a robot (even a costly humanoid) is socially and economically viable.
It is important to highlight that the introduction of humanoids in aeronautic plants should be facilitated because the aeronautic shop floor is a very well mastered environment:

- 3D models of the aircraft and the shop floors exist, enabling model-based reference and localization of the robot,

- the Airbus operators are trained: they follow strict rules and assembly sequences, which will simplify humanhumanoid interaction.

This study covers a frequent use-case encountered in production. It aims at investigating the capability (and hence the feasibility) of a humanoid to check the correct behavior of the electrical systems of the plane or helicopter once it has been assembled. Circuit breakers are used on aircrafts in order to protect an electrical circuit from damage caused by current excess. Their state can also be switched manually in order to reset operation or to switch off a function. In particular, during ground testing, operators manipulate the circuit breakers panel in order to validate the behavior of the systems in a large number of configurations.

The technological bottleneck concerns (i) navigation that we are addressing in the frame of the COMANOID ${ }^{3}$ project [8] using multi-contact technology [6], [7], [5], [9] that is yet to be demonstrated with our humanoid in a $1: 1$ scale airplane mock-up, and (ii) once near the circuit-breaker panel, achieve the functional verification tasks and eventually report any encountered problem. For this purpose, AGI has provided a mock-up that is described in section V.

\section{Multimodal task SPACE QP CONTROL}

To realize our use-case, the humanoid has to walk to the panel and stop nearby in an appropriate configuration (section IV). Then it has to lean on the panel with the (left) gripper that does not operate the switches (the reason for that and the methodology are explained in section IV), before starting to move the pulling tool attached to the (right) gripper using visual servoing in force-guarded motion, clamp the switch in the appropriate position, pull it under guarded motion and finally release the tool and start again with another switch. All these tasks have to be achieved under constraints of equilibrium, state (joint position, velocity and acceleration) limits, torque limits, non-sliding contact forces, no auto-collision, keeping the tracked marker in the field-ofview $(\mathrm{FoV})$ of the camera, etc.

Multi-objective, task-space control [10] formulated as quadratic programming appeared recently as a golden standard for whole-body control of humanoids, see examples in [11][12][13][14][15][16][17][18][5]. Tasks can be ordered in strict, weighted or a hybrid priority. In a weighted (soft) priority scheme, that we are using, the tasks to be achieved at best are weighted according to their priority and summed in the cost function part of the QP. Those objectives that need to be fulfilled strictly are in the constraint part of the QP. For example, creating a contact is split into (i) a reaching contact task and (ii) a contact task. Reaching the contact is

\footnotetext{
${ }^{3}$ www. comanoid.eu
} 
a task that is put first in the cost function. As soon as the contact is established, this task is removed and shifted into a QP constraint to maintain the contact. Tasks inclusions and removals, and changes of priority is scheduled and managed by a finite state machine, see section $\mathrm{V}$ and [5].

To be self-contained, we recall here the main ingredients of the QP control framework, with the classical tasks and the newly integrated ones: visual servoing and force control.

Each task $\mathscr{T}$ is defined as an error in the sensory space that is a function of the QP decision variable (robot state accelerations, torques, or applied forces) to which is associated:(i) a Jacobian matrix $J$, (ii) a weight that defines the soft priority $w$, and (iii) gains (namely, a stiffness $k$ ).

The most usual tasks are position-based (error between any point $p_{\text {robot }}(q)$ of the robot, like an end-effector position, center of mass (CoM) position... and a desired one). For $N_{p}$ such tasks with error $\mathscr{T}_{p}=p_{\text {robot }}-p_{\text {target }}$, and weights $w_{p}$, we write the cost function

$$
\mathscr{P}=\sum_{p=1}^{N_{p}} w_{p}\left\|\ddot{\mathscr{T}}_{p}+2 \sqrt{k_{p}} \dot{\mathscr{T}}_{p}+k_{p} \mathscr{T}_{p}\right\|
$$

with $\ddot{\mathscr{T}_{p}}=J_{p} \ddot{q}+j_{p} \dot{q}$. Tasks in position with strict inclusions, equalities and inequalities are integrated in the constraints part after two derivations that result in a linear form in the decision variables; they are gathered into $\mathscr{P}_{c}$.

$N_{v}$ visual servoing tasks are defined as residuals in the task function space $\mathscr{T}_{v}=s-s_{\text {target }}, s$ being the visual features, and $s_{\text {target }}$ their desired value. The time derivative of the residual is related to the joint velocities through the Jacobian $J_{v}=L_{v} J, L_{v}$ being the interaction matrix (i.e. the visual features Jacobian) and $J$ being that of the robot as in classical visual servoing formulation. Hence, $\dot{\mathscr{T}}_{v}=L_{v} J \dot{q}$ and its derivative $\ddot{\mathscr{T}}=\dot{L}_{v} J \dot{q}+L_{v} \dot{J}_{v} \dot{q}+L_{v} J \ddot{q}$ that is integrated in the cost function with weight $w_{v}$ and gain $k_{v}$ as

$$
\mathscr{V}=\sum_{v=1}^{N_{v}} w_{v}\left\|\ddot{\mathscr{T}}_{v}+2 \sqrt{k_{v}} \dot{\mathscr{T}}_{v}+k_{v} \mathscr{T}_{v}\right\|
$$

More details on this formulation can be found in [19]. Here also, visual tasks with strict inclusion, equalities or inequalities are integrated in the constraints part as previously and gathered in $\mathscr{V}_{c}$.

Force control at a given robot operational point is implemented as an interplay between two functions. First, the target force $f_{\text {target }}$ can be either user defined $f_{d}$ or the output $f$ of the QP controller. Let $N_{f}$, be the number of force control tasks. Since the force $f$ is a QP decision variable, if $f_{d}$ is defined, the force task writes simply as $\mathscr{T}_{f}=f-f_{d}$. In this latter case $\dot{\mathscr{T}}_{f}=\ddot{\mathscr{T}}_{f}=0$ by definition; it is the "QP force task" in Fig. 2. We also define an admittance task exactly in a form of a position-type one (previously discussed), where the $\dot{\mathscr{T}}_{f}=\dot{p}_{\text {robot }}-\dot{p}_{\text {target }}$ such that $\dot{p}_{\text {target }}=\left.K_{f}\left(f_{\text {target }}-f_{\text {sensor }}\right)\right|_{n}$. $\ddot{p}_{\text {target }}$ and $p_{\text {target }}$ are obtained by numerical derivation and integration respectively; $K_{f}$ is a gain and $n$ is the surface normal; this is the "QP admittance task" in Fig. 2. Finally,

$$
\mathscr{F}=\sum_{f=1}^{N_{f}} w_{f}\left\|\ddot{\mathscr{T}}_{f}+2 \sqrt{k_{f}} \dot{\mathscr{T}}_{f}+k_{f} \mathscr{T}_{f}\right\|
$$

It is understood that eq. (3) splits in two tasks (force and admittance in Fig. 2) when $f_{d}$ is specified, and it is only the admittance task, when $f_{d}$ is not given by the user. As for previous task-types, inclusions, inequalities and equalities can be defined as constraints gathered in $\mathscr{F}_{c}$.

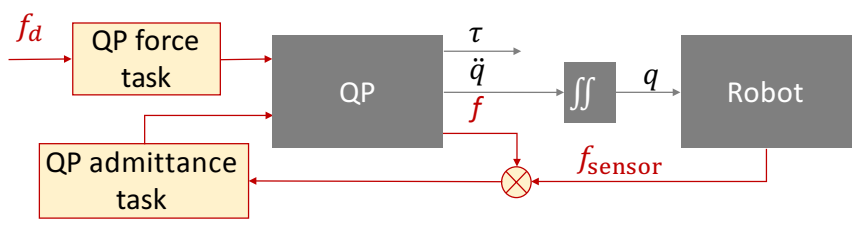

Fig. 2: Implementation of the force regulation within the QP framework.

At each control time step $t$, the controller with the set of tasks previously defined is fed back with the current state of the robot $(q, \dot{q})$ and the sensors parameters. It then solves for the decision variables: robot state acceleration $\ddot{q}$, the stacked vector of forces $f$, and the actuation torques $\tau$ through the following QP:

$$
\min _{(\ddot{q}, f, \tau)} \mathscr{P}+\mathscr{V}+\mathscr{F}
$$

subject to: $\mathscr{P}_{c}, \mathscr{V}_{c}, \mathscr{F}_{c}$, plus the common additional ones as follows:

$$
M(q) \ddot{q}+C(q, \dot{q})+G(q)=S \tau+J^{T} f
$$

the dynamic equation linking all the decision variables, with $S$ a selection matrix for the actuated joints in $q, J$ is the force to torques mapping Jacobian. $M, C$ and $G$ are the classical Inertia matrix and the Coriolis and Gravitation vectors respectively. Pre-multiplying this equation by $S^{T}$, we can express $\tau$ as a affine function of $\ddot{q}$ and $f$ and remove it from the variables.

We also have the kinematic (fixed) contact tasks as previously exemplified. Once the contact is reached, it is shifted into the constraint part of the QP through this task:

$$
J \ddot{q}+\dot{J} \dot{q}=0
$$

The torque limits task inherent from the actuators characteristics are expressed as:

$$
-\tau_{\max } \leq \tau \leq \tau_{\max }
$$

Then we have:

$$
A f \leq 0,
$$

for non-sliding contacts. This task keeps the forces $f$ within their linearized friction cones represented by $A$, see [20].

Collision avoidance is also integrated in the QP as described in [5]:

$$
\dot{d}+t \ddot{d} \geq \xi \frac{d-d_{\sigma}}{d_{i}-d_{\sigma}}
$$

where $d$ is the distance between a pair of bodies computed using [21], $d_{\sigma}$ is the threshold distance, under which we consider that collision happens; $d_{i}$ is the influence distance to activate the damping; $\xi$ is a damping coefficient and $t$ is the control time-step. 
As in [5], waypoints tasks are integrated as guide-paths to avoid local minima. They also resolve the contradiction between moving a body to a desired contact spot and at the same time avoiding collision between that body and the environment component on which the contact is defined. Note that our controller integrates in a single formulation multiple robots (i.e. any objects, including other robots, that can be represented as robotic structures even with passive joint), see [22][23].

\section{TASK-AWARE CONTACT PLANNING}

What makes humanoids a plausible perspective for their deployment in aerospace -or other large-scale productsmanufacturing, is their ability to use multi-contact technology, which has been the focus of our research and development in the past years [7][6][5], see also ${ }^{4}$. Indeed, humanoid locomotion in cluttered environment exploits possible sequence of contact creations and removals between any part of the robot and the environment to plan motions. Robotic inhand and in-body object manipulation also exploits contacts and their repositioning for assembly operations. In fact, legs can enhance manipulation capabilities and grippers can enhance the locomotion ones. The two problems interchange in sharing the same background: they are governed by nonsmooth dynamics (friction and impacts at contacts) under limiting constraints including stability. Therefore, we have considered them jointly [6].

In the context of humanoid manufacturing, multi-contact capabilities come with an extra bonus: a humanoid can plan a contact to make a closed kinematic-chains that allows it to better sustain its equilibrium when needed or to apply higher forces thanks to internal torques. Let us exemplify this bonus with our circuit breaker scenario. The humanoid stands near the panel, and then pulls each switch one after the other. To pull the switch a force of about $13 \mathrm{~N}$ is needed. Since humanoids have a floating base, the pulling force needs to be balanced by other contact forces. In preliminary experiments (section V), when the tool abruptly released the button during pulling, discontinuity in the applied force caused the robot to oscillate what could lead to balance loss if the force needed is high. Moreover, when pulling the switch (or a drawer or a door) the upper body may tend to lie toward it due to ankle flexibility if there is no postural counterbalance task.

In this example, if the humanoid put the palm of its other hand on the panel, it creates additional close kinematic chains (in addition to the existing ones due to feet contacts), which results in the robot being able to apply higher forces on the switch, generated from internal forces. It also prevents the robot from tilting toward the panel during pulling, and increases the equilibrium area and robustness.

The generalization of this concept is an on-going work that constitutes a substantial contribution to the multi-contact planning field in its own. As this contact planning is related to the task to be performed, we named it task-aware contact

\footnotetext{
${ }^{4}$ www. comanoid.eu
}

planning. We have two main ingredients to achieve this goal in this purpose study:

1) our current planning [6] and control [22] considers all manipulated objects and the environment as "robots" that are represented as a kinematic trees;

2) we recently devised a posture generator (PG) software that can compute static postures under a set of constraints including prevision on the applied forces (with equalities, bounds, inequalities) [9].

We integrate the creation of such a contact, that is forcetask driven (instead of the locomotion-driven) to the general multi-contact planning context. Indeed, to add a contact what needs to be provided to the PG is:

1) the force to be applied and its location on the humanoid robot (according to a frame attached to the robot's link that will apply that force) and also a frame where in the other robots (i.e. the environment, the object, another robot...) it operates;

2) all the remaining humanoid robot's links, that are not in contact, paired with all the surfaces, where an additional contact can be added with a resultant force, that is greater in intensity to the task one with the opposite direction.

The first step is provided by the manufacturing planner or the user. The second step boils to what is usually used in our general purpose multi-contact planning [7][6]. But considering all the contact (link, surfaces) possibilities is certainly not a viable approach, and reducing the combinatorial search space is crucial in this context.

For example, let $\mathscr{R}_{\text {env }}$ be the fixed environment, $\mathscr{R}_{\text {rob }}$ be our humanoid, and $\mathscr{R}_{\text {pan }}$ the panel system represented by $N_{b}+1$ links, where $N_{b}$ is the number of switches. In this case $N_{b}$ is also the number of prismatic joints of the panel system because the switch sockets are linked rigidly to $\mathscr{R}_{\text {pan }}$ which in turn is rigidly linked to $\mathscr{R}_{\text {env }}$. We invoke the PG with the following task constraints:

- contact $\mathscr{R}_{\text {rob }}$ 's left foot with $\mathscr{R}_{\text {env }}$ 's ground (that can be fixed or free),

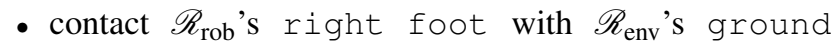
(that can be fixed or free),

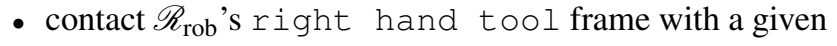
$\mathscr{R}_{\text {pan }}$ 's switch frame with $n_{t} . f_{c_{t}} \geq f_{\text {task }}$, where $n_{t}$ is the contact normal, $f_{c_{t}}$ is a decision variable of the PG and $f_{\text {task }}$ is the given (pulling) force;

- contact $\mathscr{R}_{\text {rob }}$ 's right hand palm with the $\mathscr{R}_{\text {pan's }}$ board (floating) with $\left\|n_{t} \cdot f_{c_{p}}+n_{p} \cdot f_{c_{t}}\right\|$ as a cost to minimize, $n_{p}$ is the panel normal and $f_{c_{p}}$ the contact force on the panel.

The Figs. 3 and 4 illustrate the obtained results for the panel with different switch positions. Notice that for each switch the posture returned is slightly different, which is normal. The resulting forces are rendered in red arrow (that of the left hand is shifted on the board, as otherwise it is not visible). In practice, we generated one posture at the center position and checked that all buttons are reachable by fixing the feet and the hand on the panel. Another solution, more 


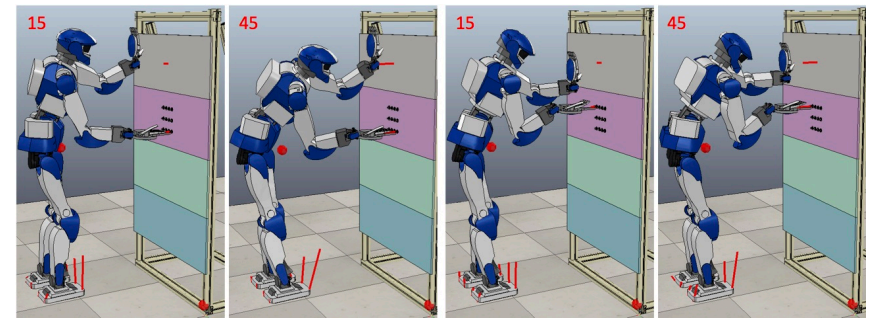

Fig. 3: Output of the PG for the pulling task of two different buttons with $f_{\text {task }}=15 \mathrm{~N}$ and $45 \mathrm{~N}$ force. The feet are set at a fixed static position w.r.t the panel. Notice the difference in posture when the pulling force is higher and the buttons are different.
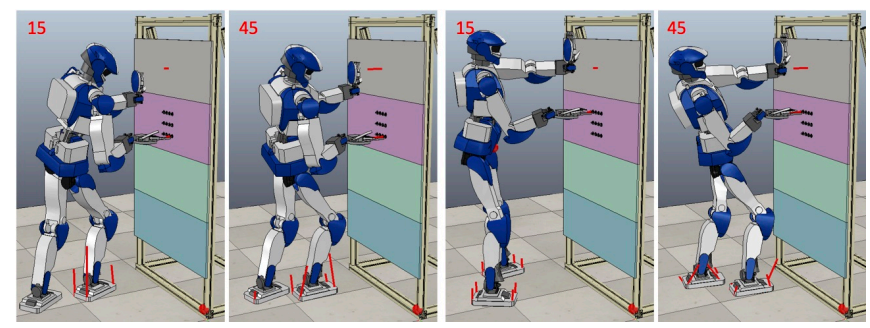

Fig. 4: Output of the PG for the pulling task of two different buttons with $f_{\text {task }}=15 \mathrm{~N}$ and $45 \mathrm{~N}$ force. The feet positioning (floating) is determined by the PG. Notice the difference in posture and feet position when the pulling force is higher and the buttons are different.

costly, is to run a robot-duplicates optimization as in [5]. We can let the positioning of the feet to the PG of fixed them (typically in cluttered spaces). It is easy to understand, that with this additional panel contact, a sudden release of the pulling force still keeps the humanoid well balanced.

\section{EXPERIMENTS AND RESULTS}

\section{A. Experimental set-up}

The humanoid used for the experiments is the HRP4. Xtion PRO LIVE camera is mounted in the head of HRP-4. It is placed in front of the panel with the circuit breakers (provided by AGI). The initial position does not have to be precisely set as planned in section IV, as visual feedback allows to perform the task successfully regardless of the variability in the starting position. Figure 5 shows one example of a feasible initial placement.

Switches on the panel are given a label based on the position in the $3 \times 4$ grid, that is $\mathrm{switch}_{i j}$, where $i$ and $j$ indicates the row and the column respectively.

Three WhyCon [24] markers are placed in the panel to form a pattern, which is not ambiguous in rotation (e.g. "L" shape). One marker is attached to the tool, such that it remains visible throughout the experiment. The pulling tool is attached to the right wrist of the robot. Example of tool and marker placement is shown in Fig. 6. The horizontal offsets from one of the markers on the panel to the switchoo are measured and later used to compute relative pose of the switch that needs to be pulled in the camera frame. Distance from the tool tip to the tool marker center $d_{\mathrm{tc}}$ is measured

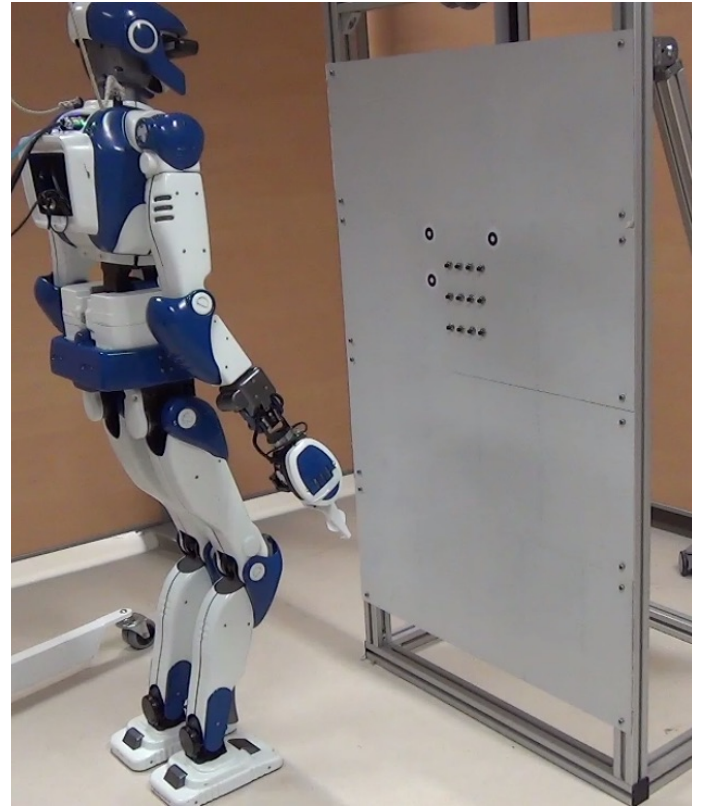

Fig. 5: Example of initial position.

and later used to set appropriate error thresholds for visual servoing tasks.

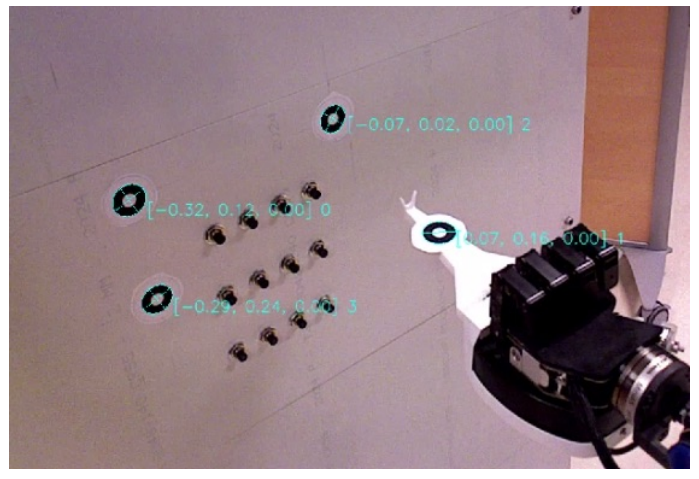

Fig. 6: Marker and tool placement.

\section{B. Finite-state machine}

The entire experiment is managed by a finite state machine (FSM) composed by a finite set of states and transitions $\left(t_{i}\right)$ between them. In each state appropriate tasks are either added to or removed from the QP. Figure 7 illustrates the FSM implementation for the circuit-breaker experiment. In the remaining part of this subsection, states and transitions of implemented FSM are described in more detail.

Initial posture is a safety state: CoM and torso orientation tasks must converge, before $t 1$ can be triggered and further tasks are then added to the QP. In hand to panel state, position task is added to move left hand (LH) to a predefined way-point. $t 2$ is triggered after position task converges. When in left hand admittance, position task for LH is removed. New contact constraint is added to ensure that left palm and panel are in contact. Admittance task with desired target force for the contact is added. $t 3$ is triggered after desired force is achieved. 


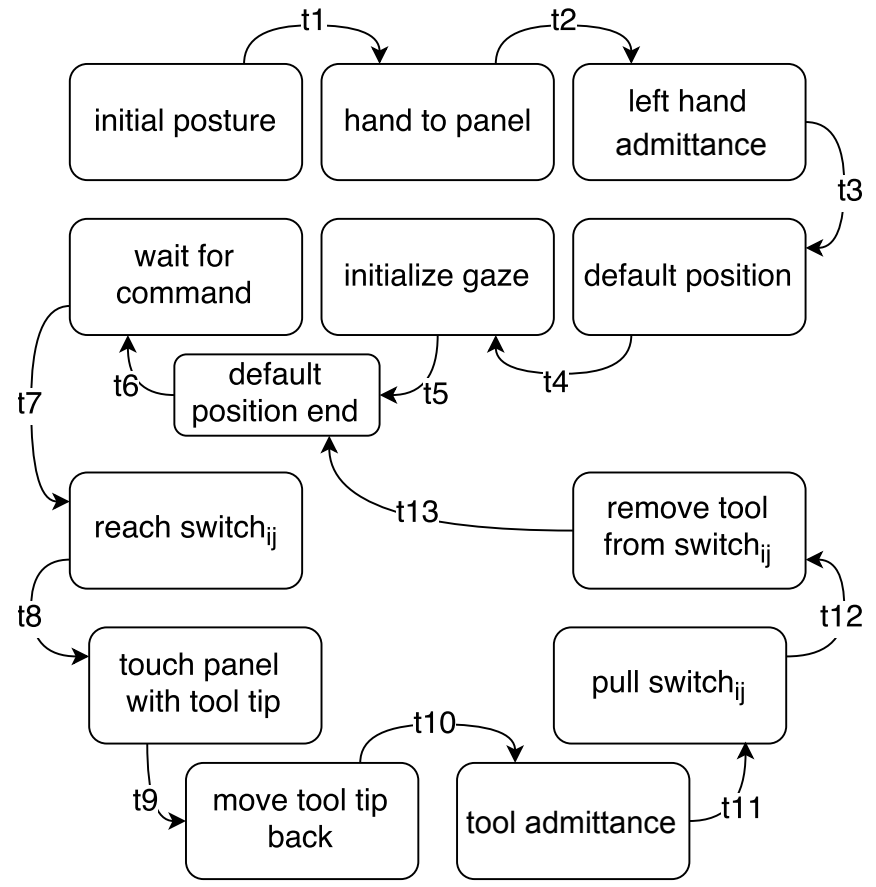

Fig. 7: FSM for circuit-breaker checking experiment.

In default position state, position task is added to move the right hand $(\mathrm{RH})$ to default position through two predefined way-points. $t 4$ is triggered once both waypoints have been reached sequentially. Image based visual servoing (IBVS) task is added in initialize gaze state, to ensure that markers appear in the FoV. Once IBVS error converges, $t 5$ is triggered. After entering default position end, position task for RH is removed. Default position of the right hand with respect to the panel is refined by adding position based visual servoing (PBVS) task. Once vision based position of the tool is less than $3 \mathrm{~mm}$ away from the target value, $t 6$ is triggered. In wait for command, robot is waiting for the command with switch label $(i j)$. After command is received, $t 7$ is triggered.

In reach $\mathrm{switch}_{i j}$, the target for PBVS task is redefined, so that the tip of the tool is positioned under $\mathrm{switch}_{i j}$ with appropriate offsets. $t 8$ is triggered once tool tip is at least $3 \mathrm{~mm}$ away from desired position. In the beginning of touch panel with tool tip PBVS task is removed. New forceguarded position task is added to detect when tool tip is in contact with the panel. When in contact (i.e. target force is reached), $t 9$ is triggered. Set point task with objective to move RH $5 \mathrm{~mm}$ back is added in move tool tip back state. $t 10$ is triggered once set point task error converges. In tool admittance, set point task is removed. New contact constraint and admittance task are added to bring tip of the tool in contact with switch $h_{i j}$. After admittance task converges, $t 11$ is triggered. In pull $\mathrm{switch}_{i j}$, set point task is added to move RH diagonally $1 \mathrm{~cm}$ up and $2.5 \mathrm{~cm}$ back to avoid tip of the tool slipping off the switch. As a result of this motion switch ${ }_{i j}$ is pulled. $t 12$ is triggered once Euclidean distance from the target position is less than $8 \mathrm{~mm}$. Contact between tool tip and the switch ${ }_{i j}$ is removed in remove tool from $\mathrm{switch}_{i j}$ state. After tool is removed away from the switch, $t 13$ is triggered and robot goes back to default position end state.

\section{Experimental results}

For the discussion of results, we indicate main phases of the experiment as follows: 1) LH position task added, 2) LH admittance task added, 3) RH position task added, 4) IBVS task added, 5) PBVS task added, 6) switch label is received, 7) pulling finished, going back to default position.

Figure 8 demonstrates changes in HRP-4 joint values. As can be seen, the joint value curves are smooth with no abrupt changes. Figures 9 and 10 illustrate evolution of task error values during the experiment. Description of the tasks with corresponding weight and stiffness values is presented in Table I. As can be seen from the plot, high weight CoM task error stays close to zero, while lower weight torso orientation task error varies. Figure 10 illustrates fast convergence of IBVS, PBVS and LH position tasks to zero. Figure 11 illustrates the $x$-component of the LH force sensor that is controlled to hold a contact on a panel with a given desired force, and RH force sensors (we plotted the norm of all force components for RH). After phase 2, LH admittance task with force target $-15 \mathrm{~N}$ is added and force value converges to this target. LH admittance task is kept in the QP until the end of the experiment, and the controller tries to satisfy the objective while other motions are being performed.

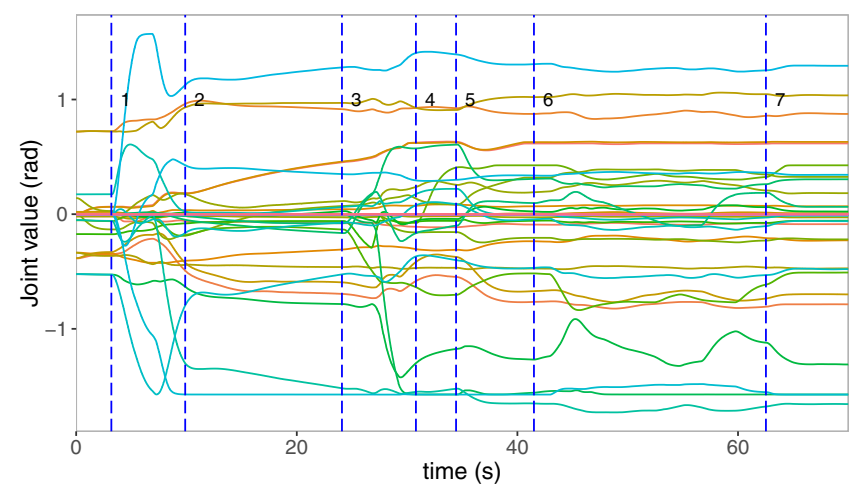

Fig. 8: All HRP-4 joint values during the experiment.

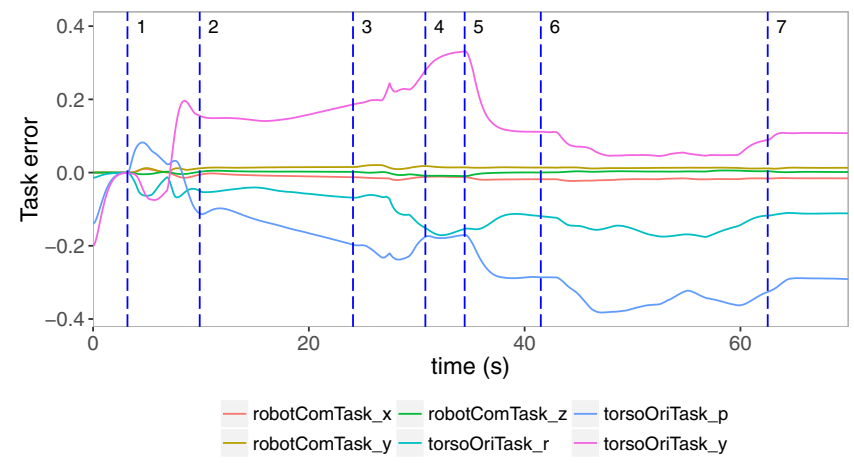

Fig. 9: General QP tasks: CoM and torso orientation task errors. 


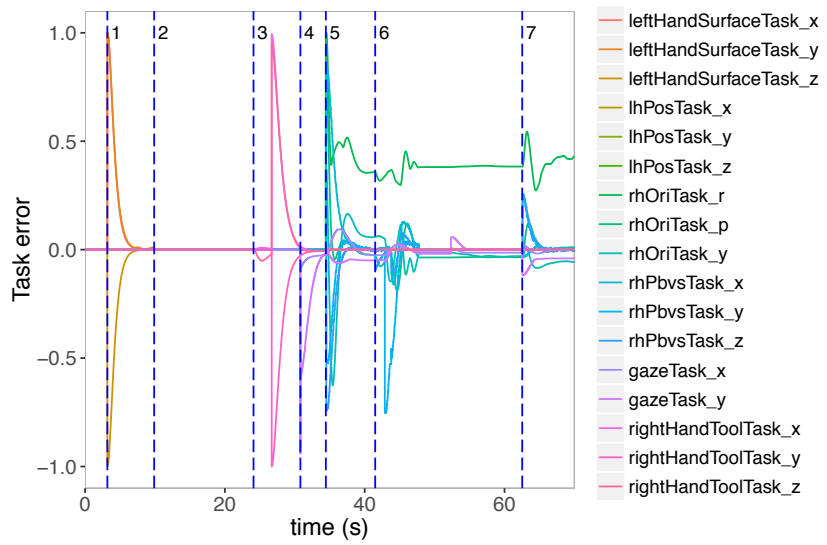

Fig. 10: Experiment specific task errors (normalized).

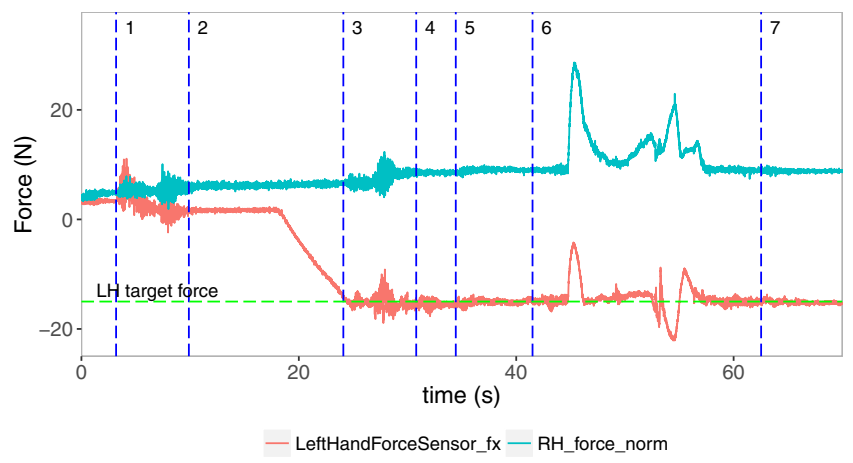

Fig. 11: The LH is controlled in admittance after phase (2) to hold a contact of $-15 \mathrm{~N}$ with the panel all along the experiment. We plotted the norm of all forces for the $\mathrm{RH}$ as in each step different components are used (guarded motion with its specific, putting the tool in the switch, pulling the switch and releasing it... all with different values of the desired force). These phases are clearly identifiable from the norm.

\section{Performance evaluation}

Stiffness of the tasks can be increased to force faster convergence of the error. However, when using visual feedback, there is a limitation on task convergence speed, due to the low frame rate of the standard camera. Task stiffness values used in QP controller are presented in Table I.

\begin{tabular}{|l|l|l|l|}
\hline \multicolumn{1}{|c|}{ Task name } & \multicolumn{1}{c|}{ Description } & Weight & Stiffness \\
\hline \hline robotComTask & Balance task & 1000 & 5 \\
\hline torsoOriTask & Torso orientation & 10 & 5 \\
\hline leftHandSurfaceTask & LH movement control & changing & 3.5 \\
\hline rightHandToolTask & RH movement control & changing & 8 \\
\hline gazeTask & IBVS task & 5 & 5 \\
\hline rhPbvsTask & PBVS RH task & 20000 & 5 \\
\hline rhOriTask & RH orientation task & 100 & 5 \\
\hline admittanceLeftHand & Target force for LH & 1000 & 8 \\
\hline admittanceTool & Target force for tool & 1000 & 8 \\
\hline rhPosTask & Avoid moving RH & 1000 & 2 \\
\hline lhPosTask & LH movement control & 10000 & 2 \\
\hline rightHandPushTask & Move tool back & 20000 & 12 \\
\hline
\end{tabular}

TABLE I: QP tasks with weight and stiffness values.

Final size of the WhyCon markers used in the experiment, is $2.4 \mathrm{~cm}$ (diameter of the outer black circle). In order to mini- mize amount of potential error in marker position estimation, maximal camera resolution was used for frame acquisition $(1280 \times 1024$ pixels $)$. This allowed to have $2-3 \mathrm{~mm}$ precision in marker localization. Calibrated camera was used to ensure low uncertainty in the camera intrinsic parameters.

\section{E. Discussions and limitations}

The experiments were successfully conducted several times in the laboratory over the course of two months without imposing any explicit constraints on the light conditions of the room (both natural and electric light were used at some point). The same controller, with some additional improvements in the implementation, was used to demonstrate this work at the first Digital Festival in Tahiti in March of 2017. It was exposed to a large audience public, and demonstration at least 6 times per day, during 2 and half days with only one failure due to a wrong operation by the user. This shows, that our experiment is highly reproducible.

We used visual markers to better test motion planing part of the controller, without depending too much in lightning conditions of the experimental setup. Several types of marker trackers have been tested for application in the experiment (e.g. ARToolKit [25] and other markers of similar type). The WhyCon marker proved to be most suitable for the application, as no false positive detection was encountered during the experiment and the accuracy of marker position estimation was sufficiently high for the task to be performed successfully. For the continuation of this work, more sophisticated solution for the visual feedback would require removing the markers from experimental setup and using visual primitives (e.g. keypoints, edges) to estimate location of the tool and the switch grid on the panel.

When estimating the state of the environment (i.e. switch and tool position) with non-maker-based computer vision methods, the amount of noise would likely increase and the smoothness of position estimation would drop. It is therefore critical to investigate the robustness of the visual servoing control w.r.t. to the measurement noise

Limitation of the speed of visual servoing task depends on the image processing operation as well as the frame rate of the camera. High values of task speed may results in convergence prior to new frame acquisition and processing. As a result, the motion of the robot would be somehow jerky.

In circuit-breaker experiment, HRP-4 right hand wrist goes very close to the joint limit value, so do other joints of the upper legs. Also high variability in initial position can cause robot to be incapable of reaching some or all of the switches on the panel. It is thus evident, that with the current platform, performing the task successfully even in the open space without clutter or obstacles, can be challenging. In aircraft manufacturing, cluttered and confined spaces are recurrent and we recommend humanoid's design with higher redundancy and also large joint ranges. The limitation to this currently is not mechanical, but has to do with the cabling.

In the current implementation of controller, the tasks gains and their weightings are set manually. Those values, however, 
are not necessarily optimal. It is crucial to automate these settings using learning techniques for instance.

\section{Conclusion}

We showed that HRP-4, controlled by multi-contact and multi-objective QP framework, can successfully perform circuit-breaker operating task. The controller design and QP tasks, including multi-modal integration of position, force and visual servoing tasks, have been presented and discussed. We demonstrate the advantage of creating closed kinematic chains as part of the multi-contact planning to increase balance robustness and task force range.

The experiment revealed that the humanoid design must have more redundancy to perform similar manufacturing tasks with higher flexibility and in more challenging environment. For the continuation of this work, non-marker-based visual feedback methods can be implemented and tested for full-body motion control in QP framework and methods to increase the execution speed can be investigated.

\section{ACKNOWLEDGEMENTS}

This work is supported in part from Airbus Group Innovation Center grants, H2020 COMANOID EU project, the CNRS-AIST-Airbus Group joint research program, the bpifrance ROMEO 2 PSPC project, the JSPS Grant-in-Aid for Scientific Research (B) Number 16H02886 ("CuttingEdge multi-contact behaviors), and the French government research program "Investissements d'avenir" through the Robotex Equipment of Excellence (ANR-10-EQPX-44) for the HRP-4 humanoid robot.

\section{REFERENCES}

[1] S. Nakaoka, S. Kajita, and K. Yokoi, "Intuitive and flexible user interface for creating whole body motions of biped humanoid robots," in Proceedings of the 2010 IEEE/RSJ International Conference on Intelligent Robots and Systems, (Taipei, Taiwan), pp. 1675-1682, 1822 October 2010.

[2] S. Nakaoka, K. Miura, M. Morisawa, F. Kanehiro, K. Kaneko, S. Kajita, and K. Yokoi, "Toward the use of humanoid robots as assemblies of content technologies -realization of a biped humanoid robot allowing content creators to produce various expressions-," Synthesiology (English edition), vol. 4, pp. 80-91, October 2011.

[3] A. Kumar Pandey, R. Gelin, R. Alami, R. Viry, A. Buendia, R. Meertens, M. Chetouani, L. Devilliers, M. Tahon, D. Filliat, Y. Grenier, M. Maazaoui, A. Kheddar, F. Lerasle, and L. F. Duval, "Romeo2 project: Humanoid robot assistant and companion for everyday life: I. situation assessment for social intelligence," in International Workshop on Artificial Intelligence and Cognition, (Torino, Italy), pp. 140-147, 26-27 November 2014.

[4] A. Paolillo, P. Gergondet, A. Cherubini, M. Vendittelli, and A. Kheddar, "Autonomous car driving by a humanoid robot," Journal of Field Robotics, to appear.

[5] J. Vaillant, A. Kheddar, H. Audren, F. Keith, S. Brossette, A. Escande, K. Bouyarmane, K. Kaneko, M. Morisawa, P. Gergondet, E. Yoshida, S. Kajita, and F. Kanehiro, "Multi-contact vertical ladder climbing with an HRP-2 humanoid," Autonomous Robots, vol. 40, no. 3, pp. 561580, 2016.

[6] K. Bouyarmane and A. Kheddar, "Humanoid robot locomotion and manipulation step planning," Advanced Robotics, vol. Special Issue on Cutting edge robotics in Japan, pp. 1099-1126, July/September 2012.

[7] A. Escande, A. Kheddar, and S. Miossec, "Planning contact points for humanoid robots," Robotics and Autonomous Systems, vol. 61, no. 5, pp. 428-442, 2013.
[8] S. Caron and A. Kheddar, "Multi-contact walking pattern generation based on model preview control of 3D COM accelerations," in IEEE-RAS International Conference on Humanoid Robots, (Cancun, Mexico), pp. 550-557, 15-17 November 2016.

[9] S. Brossette, A. Escande, and A. Kheddar, "Multi-contact postures computation on manifolds," IEEE Transactions on Robotics, submitted.

[10] C. Samson, M. Le Borgne, and B. Espiau, Robot control- the task function approach. Oxford, England: Clarendon Press, 1991.

[11] L. Sentis, J. Park, and O. Khatib, "Compliant control of mult-icontact and center-of-mass behaviors in humanoid robots," IEEE Transactions on Robotics, vol. 26, no. 3, pp. 483-501, 2010.

[12] J. Salini, S. Barthélemy, and P. Bidaud, LQP-based controller design for humanoid Whole-body motion, pp. 177-184. Springer, 2010.

[13] K. Bouyarmane and A. Kheddar, "Using a multi-objective controller to synthesize simulated humanoid robot motion with changing contact configurations," in IEEE/RSJ International Conference on Intelligent Robots and Systems, (San Fransico, CA), pp. 4414-4419, 25-30 September 2011.

[14] L. Righetti and S. Schaal, "Quadratic programming for inverse dynamics with optimal distribution of contact forces," in IEEE-RAS International Conference on Humanoid Robots, (Businness Inoovation Center, Osaka, Japan), pp. 538-543, 29 November - 1 December 2012.

[15] P. M. Wensing and D. E. Orin, "Generation of dynamic humanoid behaviors through task-space control with conic optimization," in IEEE International Conference on Robotics and Automation, (Karlsruhe, Germany), pp. 3088-3094, 6-10 May 2013.

[16] A. Escande, N. Mansard, and P.-B. Wieber, "Hierarchical quadratic programming: Fast online humanoid-robot motion generation," International Journal of Robotics Research, vol. 33, no. 7, pp. 1006-1028, 2014.

[17] S. Kuindersma, F. Permenter, and R. Tedrake, "An efficiently solvable quadratic program for stabilizing dynamic locomotion," in IEEE International Conference on Robotics and Automation, (Hong Kong, China), pp. 2589-2594, 31 May - 07 June 2014.

[18] S. Feng, E. Whitman, X. Xinjilefu, and C. G. Atkeson, "Optimizationbased full body control for the DARPA robotics challenge," Journal of Field Robotics, vol. 32, pp. 293-312, March 2015.

[19] D. J. Agravante, G. Claudio, F. Spindler, and F. Chaumette, "Visual servoing in an optimization framework for the whole-body control of humanoid robots," IEEE Robotics and Automation Letters, vol. 2, no. 2, pp. 608-615, 2017.

[20] S. Caron, Q.-C. Pham, and Y. Nakamura, "Leveraging cone double description for multi-contact stability of humanoids with applications to statics and dynamics," in Robotics: Science and System, (Roma, Italy), 13-17 July 2015.

[21] A. Escande, S. Miossec, M. Benallegue, and A. Kheddar, "A strictly convex hull for computing proximity distances with continuous gradient," IEEE Transactions on Robotics, vol. 30, pp. 666-678, 13 january 2014.

[22] J. Vaillant, K. Bouyarmane, and A. Kheddar, "Multi-character physical and behavioural interactions controller," IEEE Transactions on Visualization and Computer Graphics, vol. 23, pp. 1650-1662, June 2017.

[23] K. Bouyarmane, J. Vaillant, K. Chappellet, and A. Kheddar, "Multirobot and force task-space control with quadratic programming," https://hal.archives-ouvertes.fr/hal-01495662/, submitted.

[24] M. Nitsche, T. Krajník, P. Čížek, M. Mejail, and T. Duckett, "WhyCon: an efficient, marker-based localization system," in IEEE/RAS IROS Workshop on Open Source Aerial Robotics, (Hamburg, Germany), 28 September 2015.

[25] H. Kato and M. Billinghurst, "Marker tracking and HMD calibration for a video-based augmented reality conferencing system," in IEEE/ACM International Workshop on Augmented Reality, pp. 85-94, 1999. 